\title{
Complications of Percutaneous Renal Biopsy
}

\author{
Kenaz Bakdash, MD ${ }^{1} \quad$ Kristofer M. Schramm, MD ${ }^{1} \quad$ Aparna Annam, DO ${ }^{1}$ Matthew Brown, MD ${ }^{1}$ \\ Kimi Kondo, $\mathrm{DO}^{1}$ Jonathan D. Lindquist, $\mathrm{MD}^{1}$
}

${ }^{1}$ Division of Vascular and Interventional Radiology, Department of Radiology, University of Colorado, Anschutz Medical Center, Aurora, Colorado

\author{
Address for correspondence Jonathan D. Lindquist, MD, Division of \\ Vascular and Interventional Radiology, Department of Radiology, \\ University of Colorado, Anschutz Medical Center, Aurora, CO 80045 \\ (e-mail: jonathan.lindquist@ucdenver.edu).
}

Semin Intervent Radiol 2019;36:97-103

\begin{abstract} Keywords

- renal biopsy

- hemorrhage

- bleeding complication

- embolization

- interventional radiology

Percutaneous renal biopsy is widely used for diagnosis, prognosis, and management of nephropathies. Complications may arise after renal biopsy, most commonly in the form of bleeding. Efforts should be taken to optimize modifiable risk factors such as hypertension, thrombocytopenia, and coagulopathy prior to the procedure. Unmodifiable risk factors such as poor renal function, gender, and underlying histologic diagnosis may be used to identify high-risk patients. Delayed presentation of bleeding complications is common, and close clinical follow-up is crucial.
\end{abstract}

The percutaneous renal biopsy (PRB) has been instrumental in the diagnosis of acute and chronic kidney disease in both the native and allograft kidney since its inception in 1951 by Iversen and Brun. ${ }^{1}$ The first renal biopsies, reported in 1923 , were open surgical procedures. ${ }^{2}$ Kark and Muehrcke modified the percutaneous procedure in 1954 by placing the patient in prone position, and using an exploratory needle to localize the kidney prior to insertion of their biopsy needles. ${ }^{3,4}$ Ultrasound guidance and automatic core biopsy systems have dramatically improved tissue adequacy and safety of the procedure. The rate of severe complications leading to patient demise has decreased from 0.12 to $0.02 \%{ }^{4-6}$

The primary indications for native PRB is to evaluate proteinuria, microscopic hematuria, renal manifestations of systemic disease, and unexplained renal failure. ${ }^{5}$ The procedure is useful in diagnosis, prognosis, and management of nephropathies; however, despite technological advances, the procedure remains high risk for several reasons. Bleeding complications are common due to the vascularity of the kidney and inability to compress the biopsy location due to a deep retroperitoneal location. Patients with renal disease often have uremia-associated platelet dysfunction, hypertension, and inflammatory states which further increase risk. ${ }^{7-9}$ An in-depth review of known risk factors is presented. Accounting for these risk factors will allow clinicians to better understand and weigh the risks and benefits of the procedure for each patient.

\section{Types of Complications}

Bleeding is the most common complication of PRB. Complications not related to bleeding are much less frequent, and include pain, infection, and inadvertent injury to adjacent organs. ${ }^{10,11}$

A large meta-analysis conducted by Corapi et al surveyed 9,474 native adult ultrasound-guided renal biopsies performed with automated biopsy devices. Major complications included erythrocyte transfusion requirement $(0.9 \%)$, angiographic intervention $(0.6 \%)$, nephrectomy $(0.01 \%)$, bladder obstruction $(0.3 \%)$, and death $(0.02 \%)$. Transient gross hematuria $(3.5 \%)$ was the only minor complication reported. ${ }^{12}$

Microscopic hematuria occurs in virtually all patients, ${ }^{4}$ and is not considered a complication. Other reported complications include perinephric hematoma and arteriovenous fistula. ${ }^{13-16}$

A large retrospective review conducted by Lees et al examined 2,563 percutaneous renal biopsies performed over 15 years. ${ }^{17}$ The rate of major bleeding was $2.2 \%$, with $1.8 \%$ of patients requiring transfusion. Angiographic intervention was required in $0.4 \%$ of cases, and $0.04 \%$ of patients died.
Issue Theme Management of Complications in IR; Guest Editor, Robert K. Ryu, MD, FSIR
Copyright (c) 2019 by Thieme Medical Publishers, Inc., 333 Seventh Avenue, New York, NY 10001, USA. Tel: +1(212) 584-4662.
DOI https://doi.org/ 10.1055/s-0039-1688422. ISSN 0739-9529. 


\section{Timing of Complications}

Delayed bleeding complications are common after renal biopsies ( - Fig. 1). A series of 750 adult patients who underwent native renal biopsy demonstrated only $67 \%$ of complications were apparent at 8 hours. Ninety-one percent of major complications had presented at 24 hours; the remaining $9 \%$ of major complications were discovered between 48 and 120 hours after the procedure. ${ }^{14}$ Another more recent series demonstrated similar results in native and transplant PRB, with $91 \%$ of major complications occurring within 12 hours after biopsy, and $98.4 \%$ within 24 hours. ${ }^{18}$ Based on these data, it is standard practice at many institutions to monitor native renal biopsy patients for 24 hours postprocedure.

Postbiopsy renal ultrasound has been studied as a tool to risk stratify patients. Waldo et al evaluated 162 patients after native PRB with a 1 hour postbiopsy ultrasound, demonstrating that the presence of a hematoma 1 hour after biopsy had a $23 \%$ positive predictive value (PPV) and a $98 \%$ negative pre- dictive value (NPV) for any major complication. Furthermore, postbiopsy hematoma greater than $3 \mathrm{~cm}$ in size demonstrated $44 \%$ PPV and $97 \%$ NPV for major complication. ${ }^{19}$

\section{Risk Factors}

\section{Hypertension}

Systolic hypertension and diastolic hypertension are important risk factors for development of bleeding complications after PRB. ${ }^{7,8,20,21}$ Corapi et al's meta-analysis did not demonstrate a statistically significant impact of systolic blood pressure (SBP) on rates of postbiopsy transfusions, but was not designed to do so. The included studies employed a heterogeneous approach to percutaneous renal biopsies with no standardization of variables. Some excluded patients with SBP greater than $140 \mathrm{~mm} \mathrm{Hg}$, and there was no uniform definition of hypertension., ${ }^{7}, 12$

Shidham et al retrospectively reviewed 645 native renal biopsies and found systolic hypertension, diastolic
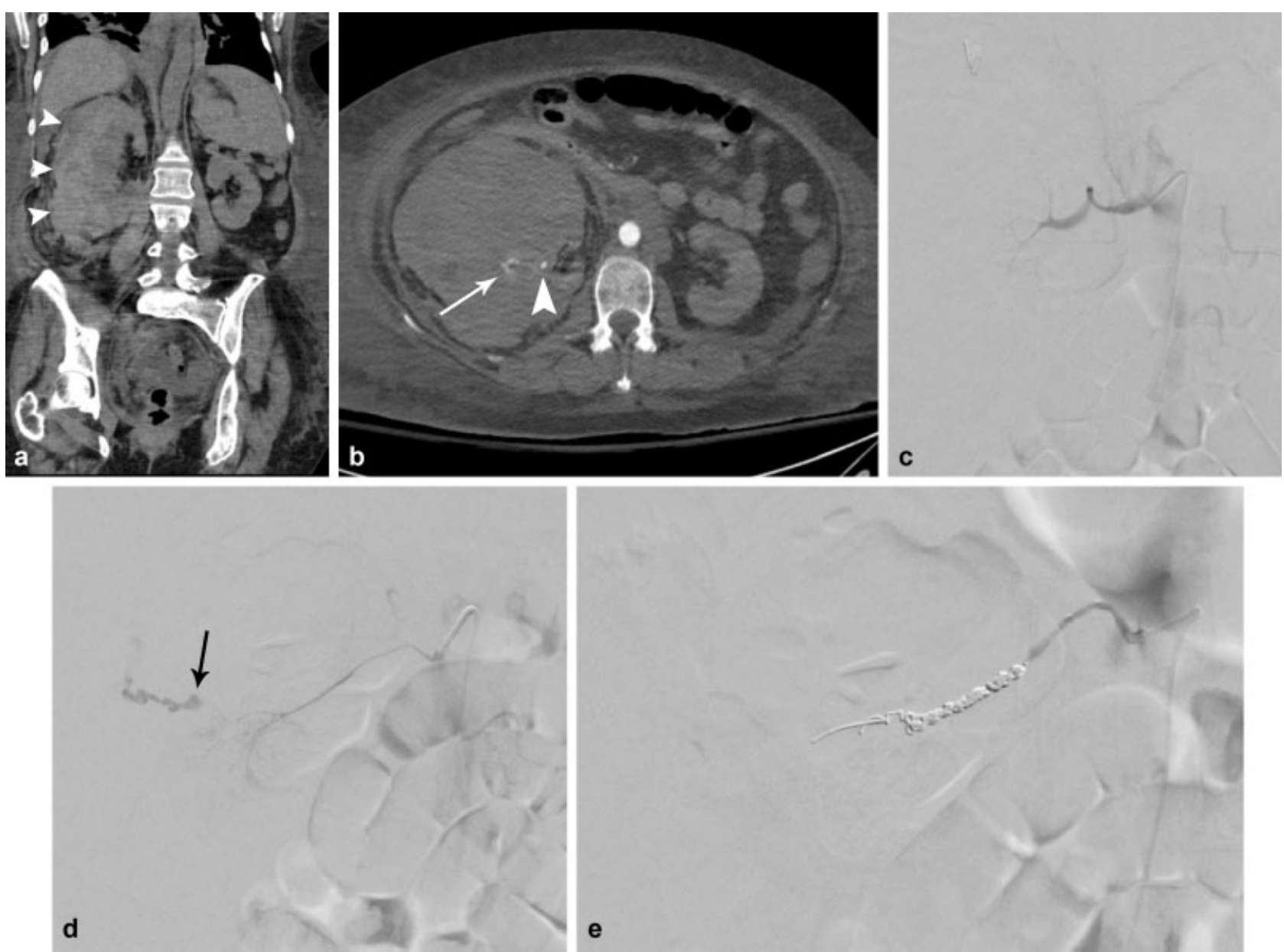

e

Fig. 1 A 51-year-old woman with nephrotic proteinuria and acute kidney injury on chronic kidney disease who was sent for native renal biopsy. Risk factors at the time of biopsy included inpatient status, elevated creatinine, anemia, thrombocytopenia, hypertension (controlled on medications), and mildly elevated INR. A 17-gauge coaxial biopsy cannula was placed. Biopsy was cancelled by the referring service midprocedure, and no cores were taken. (a) Coronal noncontrast CT 10 days after aborted biopsy for acute hemoglobin drop demonstrates large right renal subcapsular hematoma (arrowheads). (b) CT angiography confirms pseudoaneurysm (arrowhead) and active extravasation (arrow). (c) Digital subtraction right renal arteriogram demonstrates focal intrarenal arterial vasospasm. No pseudoaneurysm or extravasation is visualized. (d) Digital subtraction inferior pole accessory renal arteriogram demonstrates active extravasation (arrow). (e) Digital subtraction angiography following coil and microvascular plug embolization of the accessory right renal artery. No further extravasation is present. The patient died from respiratory failure and acute blood loss anemia the following day. 
hypertension, and elevated mean arterial pressure (MAP) to all be significant risk factors for bleeding complications. ${ }^{8}$ SBP greater than $160 \mathrm{~mm} \mathrm{Hg}$ carried a $10.7 \%$ risk of major bleeding complication, compared with 5.3\% risk if SBP was less than $160 \mathrm{~mm} \mathrm{Hg}$. Diastolic blood pressure (DBP) greater than $100 \mathrm{~mm} \mathrm{Hg}$ and MAP greater than $120 \mathrm{~mm} \mathrm{Hg}$ similarly carried greater than twice the risk of bleeding complications.

A recent retrospective study by Kriegshauser et al showed that patients with SBP greater than $140 \mathrm{~mm} \mathrm{Hg}$ and DBP greater than $90 \mathrm{~mm} \mathrm{Hg}$ experienced major bleeding complications 10 times more frequently than their nonhypertensive counterparts. When analyzed further, patients with SBP greater than $170 \mathrm{~mm} \mathrm{Hg}$ were found to experience major complications 23 times more frequently, and patients with a SBP between 141 and $170 \mathrm{~mm} \mathrm{Hg} 7$ times more frequently. Patients with DBP greater than $90 \mathrm{~mm} \mathrm{Hg}$ were also found to experience major complications seven times as frequently. ${ }^{7}$ These results are in line with other, more recent studies, although the magnitude of the resulted odds ratio (OR) in SBP greater than $170 \mathrm{~mm} \mathrm{Hg}$ is unique to this study. ${ }^{6,22}$

The effect of immediate prebiopsy reduction of blood pressure in hypertensive patients to a normotensive level has not been studied. Some authors have suggested that increased risk of postbiopsy hemorrhage could be related to limited ability of arterioles to contract as a result of arteriolar hyalinosis. ${ }^{8,9}$ Many large biopsy reviews exclude hypertensive patients, or do not standardize periprocedural blood pressure management. Though further investigation is needed in this area, periprocedural blood pressure management is standard of practice at our institution with goal of less than 140/90 $\mathrm{mm} \mathrm{Hg}$.

\section{Kidney Function}

Poor kidney function has been associated with an increased risk of major complications in several studies. ${ }^{6,12,15,23}$ Korbet et al found almost a twofold increase in rates of postbiopsy transfusion for patients with a serum creatinine greater than $3.5 \mathrm{mg} / \mathrm{dL}^{.}{ }^{6}$ The meta-analysis of Corapi et al demonstrated a higher risk for postbiopsy transfusion in patients with a serum creatinine greater than $2 \mathrm{mg} / \mathrm{dL}(2.1$ vs. $0.4 \%$, $p=0.02){ }^{12}$ In a large Chinese biopsy series by Xu et al examining 3,577 native PRBs, each $1 \mathrm{~mL} /$ minute $/ 1.73 \mathrm{~m}^{2}$ increase in glomerular filtration rate (GFR) was associated with a $4 \%$ decrease of severe bleeding risk. ${ }^{15}$ Whittier et al also demonstrated an increased risk of both minor and major complications in patients with higher serum creatinine values ( 3.5 vs. $2.2 \mathrm{mg} / \mathrm{dL}$ ). ${ }^{23}$

\section{Thrombocytopenia and Platelet Dysfunction}

The studies that have been conducted to determine if recent antiplatelet therapy prior to PRB is a risk factor for postbiopsy complications have been retrospective in nature, and no consensus regarding their periprocedural use has been reached. ${ }^{17}$ Current Society of Interventional Radiology (SIR) recommendations are to hold aspirin and clopidogrel for 5 days prior to procedures with significant bleeding risk. ${ }^{24}$ It is well known that patients with chronic renal disease have significant cardiovascular morbidity, and patients at risk for coronary artery disease have demonstrated a 3-fold increase of major adverse cardiac events after cessation of aspirin, and an 89-fold increase in patients with intracoronary stents. ${ }^{17,25}$ Additionally, these patients may have stents or grafts with a high risk of thrombosis that necessitates continuous antiplatelet therapy.

Several studies have examined the use of antiplatelet medications in the periprocedural period. Corapi et al's meta-analysis showed no difference in transfusion rate based on whether aspirin was held. ${ }^{12}$ Another large series by Lees et al showed no significant increase in major complications based on whether aspirin or clopidogrel was held. ${ }^{17}$ Baffour et al studied the effects of antiplatelet therapy on major complications in renal allograft biopsies and analyzed results based on amount of time antiplatelet therapy had been discontinued (0-3 vs. $4-7$ vs. $8-10$ days) and also found no statistically significant increase in major bleeding complications. There was, however, a sixfold increase in major complications seen in patients who had taken $325 \mathrm{mg}$ of aspirin within 3 days of biopsy (OR, 6.3; 95\% CI, 1.27-31.3). ${ }^{26}$ Mackinnon et al also suggested no difference in major complication rate with aspirin continuation. ${ }^{27}$ Pieper et al examined the effects of recent clopidogrel use in 69 patients on major complications after percutaneous core biopsy, with a mean time of clopidogrel abstinence before biopsy being 2.9 days \pm 1.9 days and found no major complications after PRB. ${ }^{28}$ However, one major limitation of these studies is the low absolute number of major complications in each study. Though this remains controversial, these studies support periprocedural continuation of aspirin in patients who are at high cardiovascular risk.

While antiplatelet therapy is controversial, thrombocytopenia has been established as an independent risk factor for postbiopsy hemorrhagic complications. SIR recommendations for periprocedural management of coagulation status recommends transfusing patients with platelets less than $50,000 / \mu \mathrm{L} .{ }^{24}$ No studies have systematically evaluated PRBs performed in patients less than this threshold, and most series excluded patients with platelet counts less than $100,000 / \mu \mathrm{L} .{ }^{10}$ Nevertheless, two studies have demonstrated an increased risk for major complication after PRB: Xu et al observed a sixfold increased rate of major complications and Torres Muñoz et al demonstrated a seven times increased risk of major complications in patients with baseline platelet counts $\leq 120,000 / \mu$ L. $^{15,22}$

Platelet function is as important as platelet count. Uremia is known to cause platelet dysfunction with elevated BUN levels greater than $60 \mathrm{mg} / \mathrm{dL}$, increasing the risk of major complications postbiopsy by up to nine times. ${ }^{22}$ Desmopres$\sin$ (DDAVP) is a synthetic antidiuretic hormone analog, and has been shown to improve hemostasis by increasing available von Willebrand factor and factor VIII levels, and decreases bleeding time in uremic patients. ${ }^{29-31}$ Manno et al conducted a prospective randomized placebo-controlled trial evaluating desmopressin as a means of reducing bleeding complications in renal biopsy patients. The study was conducted in normotensive patients with normal creatinine levels. Preprocedural desmopressin administration 
significantly reduced bleeding complications and hematoma size when compared with placebo. ${ }^{29}$

\section{Coagulopathy}

Coagulopathy should be reversed prior to PRB. To our knowledge, no studies have specifically examined PRB in the coagulopathic patient. Coagulopathy is uniformly recognized as a risk factor for major complications, and current SIR guidelines recommend correction of intrinsic and extrinsic pathway derangements prior to undergoing PRB. INR should be $<1.5$ and heparin should be held or reversed for aPTT values $>1.5$ times control. Novel anticoagulants should be held according to guidelines. ${ }^{24}$

\section{Gender}

Several studies have suggested women are at an increased risk of major complications related to PRB. ${ }^{23,32,33}$ Manno et al demonstrated that women were twice as likely to encounter a major complication after PRB compared with men. ${ }^{32}$ In a study of hospitalized patients, women were found to have between an eight- to ninefold chance of encountering a major complication. $^{33}$ The reason for this increased risk is unknown, but is postulated that it may be due to women having lower GFRs for the same serum creatinine in men, higher rates of anemia, and smaller kidney size with higher potential of deep penetration of the biopsy needle. ${ }^{12,23}$

\section{Age}

Kidney disease is common in the elderly (>60 years of age).$^{10}$ Older age at the time of biopsy did not demonstrate a statistically significant increase in major complications postbiopsy in large reported series. ${ }^{7,12,14-16}$ However, older patients who were biopsied in an emergent setting had an increased risk of major complications. ${ }^{17}$

\section{Biopsy Needle}

Biopsy needle choice has been shown to impact major complication rate. The most commonly used automated needle sizes during PRB are 14-, 16-, or 18-gauge. ${ }^{10}$ Larger needle gauge has been shown to have increased major complication rate. ${ }^{12,19}$ Whittier et al demonstrated an equivalent diagnostic accuracy of 14- and 16-gauge needles, implying a larger needle is not necessary. ${ }^{23} \mathrm{~A}$ smaller gauge needle may lead to an increased pass count, though no correlation with pass count and increased complications has been identified. ${ }^{12,15}$ Constantin et al compared 16-gauge end-cut needles with 14-gauge side-cut needles, and found no difference in diagnostic yield with fewer major complication in the 16-gauge group. Of interest, the 16-gauge end-cut needle required fewer passes than the larger side-cut needle. ${ }^{34}$ Additionally, Hergesell et al demonstrated that even with an 18-gauge needle, $99 \%$ of samples were adequate for pathologic diagnosis. ${ }^{35}$ These studies demonstrate that the use of smaller needles may decrease the risk of major complications postbiopsy. In our practice, 18-gauge BioPince needles are used for renal biopsies (Argon Medical Devices, Frisco, TX), as they yield full-thickness core specimens, as opposed to comparable side-cut devices. Two passes are typically required to acquire an adequate number of glomeruli.

Regarding coaxial and non-coaxial technique, Babaei Jandaghi et al compared 18-gauge coaxial and non-coaxial needles. Coaxial technique was found to have significantly shorter procedural time, fewer rates of complications (all minor), and increased number of obtained glomerular profiles. $^{36}$

\section{Histologic Diagnosis}

Underlying pathology in the kidney impacts risk of biopsyrelated complications. Fisi et al presented a series of 353 patients, and have demonstrated significant differences in complication rates based on underlying pathology. Pathologies such as diabetic nephropathy and acute tubular necrosis have relatively low risk for bleeding complications, while patients with thin basement membrane syndrome have six times higher than average risk. Vasculitis, rapidly progressive glomerulonephritis, and acute interstitial nephritis have twice the risk of arteriovenous fistulae, while atherosclerosis is actually protective against development of fistulae. ${ }^{37}$ Biopsy results may be useful in identifying patients at risk for delayed complications.

\section{Special Populations}

\section{Elective versus Emergent}

PRB is utilized in emergent situations where diagnosis cannot be made by laboratory tests alone. The emergent nature of a PRB is a risk factor for major complications postbiopsy. It is thought that these patients are at an increased risk of major complication because the acuity of their illness and urgency for obtaining the biopsy may preclude the ordering provider from fully correcting coagulopathies or adequately lowering blood pressure. ${ }^{12}$

Lees et al demonstrated an increased risk for bleeding in emergent versus elective PRB (3.4 vs. 1.1\%). The patients requiring emergent renal biopsy were also older, with worse renal function and higher degree of proteinuria. ${ }^{17}$ This is expected given increased rates of major complications with poor renal function. Interestingly, complications were discovered later in the emergent and hospitalized population, with $14 \%$ of complications discovered after 24 hours. $^{23}$

Moledina et al studied 159 hospitalized patients with AKI over the course of 2 years, comparing rates of complications between these patients and nonhospitalized patients from the prospective Yale University biopsy cohort. The hospitalized cohort had a higher major complication rate, with $8 \%$ requiring a blood transfusion and $2 \%$ requiring angiographic intervention. ${ }^{33}$ Additionally, postbiopsy blood transfusions were more frequent in hospitalized women, intensive care unit patients, patients with higher BUN ( $>60 \mathrm{mg} / \mathrm{dL}$ ), lower baseline hemoglobin $(<10 \mathrm{~g} / \mathrm{dL}$,), lower platelets $(<112,000 / \mu \mathrm{L})$, or had a 16-gauge needle as opposed to an 18-gauge needle used during the procedure. ${ }^{33}$ Similarly, Korbet et al found increased serum creatinine, decreased hemoglobin level, female gender, and increased SBP all to be predictive for complications in the setting of $\mathrm{AKI}{ }^{38}$ 
These findings are not simply the result of increased surveillance in the inpatient setting. These studies demonstrate that even among an increasingly surveilled group, patients with the above risk factors still demonstrated the need for more transfusions.

\section{Renal Allografts}

Renal transplant patients undergo PRB for two general reasons. Routine "protocol" biopsies are performed for early detection of allograft rejection. PRB are also performed for "cause" when specific clinical signs of rejection or allograft dysfunction is present (- Fig. 2). ${ }^{39}$ Risk of complications vary based on indication for allograft biopsy.

Morgan et al retrospectively analyzed 2,514 allograft renal biopsies. Overall major complication rate was $1.9 \%$, and included hospitalization, transfusion, operative exploration, and angiographic intervention. Risk factors for major complications were significantly higher in the "cause" group than in the "protocol" group (2.7 vs. $0.33 \%$ ). Additional risk factors included increasing age, BUN, lower platelet counts, and patients who had received deceased donor allografts. ${ }^{39}$

Redfield et al studied 3,738 renal allograft PRBs performed with an 18-gauge automated needle. The rates of severe and life-threatening complications were found to be 0.21 and $0.19 \%$, respectively, with the majority of serious complications presenting after 4 hours postprocedure. PRB within 1 week of transplant increased risk of complication by an astounding $311 \% .{ }^{40}$
Whittier et al directly compared complication rates and complication risk factors in native PRB $(n=767)$ and allograft "cause" PRB $(n=938)$ from within the same patient cohort. Compared with the native PRB cohort, allograft PRB cohort had a higher SBP, serum creatinine, and lower baseline hemoglobin. Although they appeared sicker, the renal allograft patients had a lower rate of major complications. This difference may be related to differences in technique between native and allograft biopsies. ${ }^{23}$ Of note, direct manual pressure can be applied to an allograft kidney due to its location, potentially tamponading a hemorrhage that otherwise would continue unabated in the retroperitoneum.

\section{Pregnancy}

Pregnant patients with unexplained renal failure, symptomatic nephrotic syndrome, lupus nephritis, or those with possible preeclampsia may undergo renal biopsy for confirmatory diagnosis. ${ }^{10,41}$ Increased renal blood flow and anatomical distortion due to a gravid uterus may inherently place pregnant patients at increased risk for complications after PRB. A systematic review by Piccoli et al reported that PRB changed management in 66\% of pregnant patients with a $2 \%$ major complication rate (blood transfusions) observed in 197 biopsies. ${ }^{42}$

\section{Obesity}

The prevalence of worldwide obesity has doubled and obesity-related health problems now affect more than 2 billion people. Over the past two and a half decades, there has been a
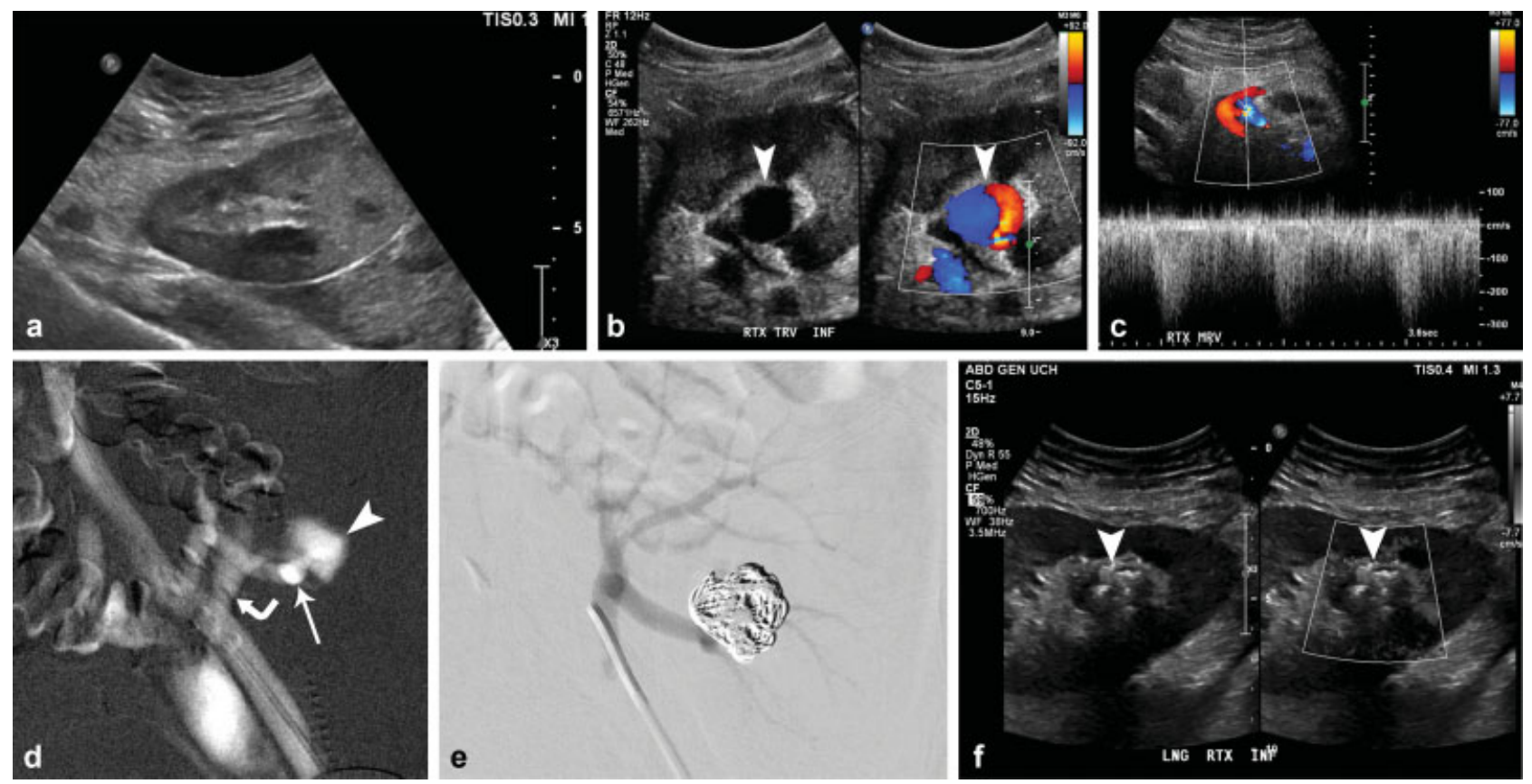

Fig. 2 Bleeding postbiopsy in a renal allograft. Biopsy was taken 2 weeks after living related transplantation to evaluate for rejection. Risk factors at the time of biopsy included systolic and diastolic hypertension (150/96 mm Hg), aspirin therapy, and elevated blood urea nitrogen (50 mg/dL). (a) Ultrasound-guided biopsy of the lower pole of the renal allograft. Three passes were required to obtain two 18-gauge cores. (b) Grayscale and color Doppler ultrasound demonstrates $2.4 \mathrm{~cm}$ renal hilar pseudoaneurysm (arrowheads) 1 year after biopsy. (c) Spectral Doppler ultrasound shows arterialized waveform within the transplant renal vein consistent with arteriovenous shunt. (d) Carbon dioxide arteriogram confirms transplant renal pseudoaneurysm (arrowhead) and arteriovenous fistula. Transplant renal artery (arrow), early filling of transplant renal vein (curved arrow). (e) Successful coil embolization with no filling of the pseudoaneurysm or arteriovenous shunting. (f) Postembolization surveillance ultrasound with no detectable color signal within the coiled pseudoaneurysm (arrowheads). 
relative increase of $28.3 \%$ in the rate of global mortality related to high BMI. ${ }^{43}$ With the rise of global obesity, the rate of obese patients requiring a PRB has also increased. Ultrasound visualization of kidneys can be technically difficult in obese patients. Interestingly, studies evaluating the rates of major complications in obese patients have shown no significantly increased risk of major complications in obese patients after PRB compared with nonobese patients. ${ }^{17,22}$ Further study of this specific population is needed to fully determine if obesity is a significant risk factor.

\section{Conclusion}

Percutaneous renal biopsy is a crucial tool for diagnosis and management of the patient with renal dysfunction. Preprocedural management of hypertension and coagulopathy decreases bleeding complications. Patients who are acutely ill and those with poor renal function are at increased risk. Selection of a smaller gauge biopsy needle decreases risk with little effect on biopsy yield. ${ }^{12,19,35}$ Monitoring native renal biopsy patients 24 hours postprocedure is warranted given the high risk of delayed bleeding complications.

Conflicts of Interest

The authors have nothing to disclose.

\section{References}

1 Iversen P, Brun C. Aspiration biopsy of the kidney. Am J Med 1951; 11(03):324-330

2 Gwyn NB. Biopsies and the completion of certain surgical procedures. Can Med Assoc J 1923;13(11):820-823

3 Kark RM, Muehrcke RC. Biopsy of kidney in prone position. Lancet 1954;266(6821):1047-1049

4 Madaio MP. Renal biopsy. Kidney Int 1990;38(03):529-543

5 Korbet SM. Percutaneous renal biopsy. Semin Nephrol 2002;22 (03):254-267

6 Korbet SM, Volpini KC, Whittier WL. Percutaneous renal biopsy of native kidneys: a single-center experience of 1,055 biopsies. Am J Nephrol 2014;39(02):153-162

7 Kriegshauser JS, Patel MD, Young SW, Chen F, Eversman WG, Chang YH. Risk of bleeding after native renal biopsy as a function of preprocedural systolic and diastolic blood pressure. J Vasc Interv Radiol 2015;26(02):206-212

8 Shidham GB, Siddiqi N, Beres JA, et al. Clinical risk factors associated with bleeding after native kidney biopsy. Nephrology (Carlton) 2005;10(03):305-310

9 McWilliams JP, Kee ST. Native renal biopsy: the perfect storm. J Vasc Interv Radiol 2015;26(02):212-213

10 Hogan JJ, Mocanu M, Berns JS. The native kidney biopsy: update and evidence for best practice. Clin J Am Soc Nephrol 2016;11 (02):354-362

11 Tabatabai S, Sperati CJ, Atta MG, et al. Predictors of complication after percutaneous ultrasound-guided kidney biopsy in HIVinfected individuals: possible role of hepatitis C and HIV coinfection. Clin J Am Soc Nephrol 2009;4(11):1766-1773

12 Corapi KM, Chen JL, Balk EM, Gordon CE. Bleeding complications of native kidney biopsy: a systematic review and meta-analysis. Am J Kidney Dis 2012;60(01):62-73

13 Marwah DS, Korbet SM. Timing of complications in percutaneous renal biopsy: what is the optimal period of observation? Am J Kidney Dis 1996;28(01):47-52
14 Whittier WL, Korbet SM. Timing of complications in percutaneous renal biopsy. J Am Soc Nephrol 2004;15(01):142-147

$15 \mathrm{Xu}$ DM, Chen M, Zhou FD, Zhao MH. Risk factors for severe bleeding complications in percutaneous renal biopsy. Am J Med Sci 2017;353(03):230-235

16 Eiro M, Katoh T, Watanabe T. Risk factors for bleeding complications in percutaneous renal biopsy. Clin Exp Nephrol 2005;9(01):40-45

17 Lees JS, McQuarrie EP, Mordi N, Geddes CC, Fox JG, Mackinnon B. Risk factors for bleeding complications after nephrologist-performed native renal biopsy. Clin Kidney J 2017;10(04):573-577

18 Prasad N, Kumar S, Manjunath R, et al. Real-time ultrasoundguided percutaneous renal biopsy with needle guide by nephrologists decreases post-biopsy complications. Clin Kidney J 2015;8 (02):151-156

19 Waldo B, Korbet SM, Freimanis MG, Lewis EJ. The value of postbiopsy ultrasound in predicting complications after percutaneous renal biopsy of native kidneys. Nephrol Dial Transplant 2009;24 (08):2433-2439

20 Diaz-Buxo JA, Donadio JV Jr. Complications of percutaneous renal biopsy: an analysis of 1,000 consecutive biopsies. Clin Nephrol 1975;4(06):223-227

21 Christensen J, Lindequist S, Knudsen DU, Pedersen RS. Ultrasound-guided renal biopsy with biopsy gun technique-efficacy and complications. Acta Radiol 1995;36(03):276-279

22 Torres Muñoz A, Valdez-Ortiz R, González-Parra C, Espinoza-Dávila E, Morales-Buenrostro LE, Correa-Rotter R. Percutaneous renal biopsy of native kidneys: efficiency, safety and risk factors associated with major complications. Arch Med Sci 2011;7(05):823-831

23 Whittier WL, Gashti C, Saltzberg S, Korbet S. Comparison of native and transplant kidney biopsies: diagnostic yield and complications. Clin Kidney J 2018;11(05):616-622

24 Patel IJ, Davidson JC, Nikolic B, et al; Standards of Practice Committee, with Cardiovascular and Interventional Radiological Society of Europe (CIRSE) Endorsement. Consensus guidelines for periprocedural management of coagulation status and hemostasis risk in percutaneous image-guided interventions. J Vasc Interv Radiol 2012;23(06):727-736

25 Biondi-Zoccai GG, Lotrionte M, Agostoni P, et al. A systematic review and meta-analysis on the hazards of discontinuing or not adhering to aspirin among 50,279 patients at risk for coronary artery disease. Eur Heart J 2006;27(22):2667-2674

26 Baffour FI, Hickson LJ, Stegall MD, et al. Effects of aspirin therapy on ultrasound-guided renal allograft biopsy bleeding complications. J Vasc Interv Radiol 2017;28(02):188-194

27 Mackinnon B, Fraser E, Simpson K, Fox JG, Geddes C. Is it necessary to stop antiplatelet agents before a native renal biopsy? Nephrol Dial Transplant 2008;23(11):3566-3570

28 Pieper M, Schmitz J, McBane R, et al. Bleeding complications following image-guided percutaneous biopsies in patients taking clopidogrel-a retrospective review. J Vasc Interv Radiol 2017;28(01):88-93

29 Manno C, Bonifati C, Torres DD, Campobasso N, Schena FP. Desmopressin acetate in percutaneous ultrasound-guided kidney biopsy: a randomized controlled trial. Am J Kidney Dis 2011;57(06):850-855

30 Franchini M. The use of desmopressin as a hemostatic agent: a concise review. Am J Hematol 2007;82(08):731-735

31 Mannucci PM, Remuzzi G, Pusineri F, et al. Deamino-8-D-arginine vasopressin shortens the bleeding time in uremia. $\mathrm{N}$ Engl J Med 1983;308(01):8-12

32 Manno C, Strippoli GF, Arnesano L, et al. Predictors of bleeding complications in percutaneous ultrasound-guided renal biopsy. Kidney Int 2004;66(04):1570-1577

33 Moledina DG, Luciano RL, Kukova L, et al. Kidney biopsy-related complications in hospitalized patients with acute kidney disease. Clin J Am Soc Nephrol 2018;13(11):1633-1640

34 Constantin A, Brisson M-L, Kwan J, Proulx F. Percutaneous US-guided renal biopsy: a retrospective study comparing the 16-gauge end-cut and 14-gauge side-notch needles. J Vasc Interv Radiol 2010;21(03): 357-361 
35 Hergesell O, Felten H, Andrassy K, Kühn K, Ritz E. Safety of ultrasound-guided percutaneous renal biopsy-retrospective analysis of 1090 consecutive cases. Nephrol Dial Transplant 1998;13 (04):975-977

36 Babaei Jandaghi A, Lebady M, Zamani A-A, Heidarzadeh A, Monfared A, Pourghorban R. A randomised clinical trial to compare coaxial and noncoaxial techniques in percutaneous core needle biopsy of renal parenchyma. Cardiovasc Intervent Radiol 2017;40 (01):106-111

37 Fisi V, Mazák I, Degrell P, et al. Histological diagnosis determines complications of percutaneous renal biopsy: a single-center experience in 353 patients. Kidney Blood Press Res 2012;35(01):26-34

38 Korbet SM, Gashti CN, Evans JK, Whittier WL. Risk of percutaneous renal biopsy of native kidneys in the evaluation of acute kidney injury. Clin Kidney J 2018;11(05):610-615
39 Morgan TA, Chandran S, Burger IM, Zhang CA, Goldstein RB. Complications of ultrasound-guided renal transplant biopsies. Am J Transplant 2016;16(04):1298-1305

40 Redfield RR, McCune KR, Rao A, et al. Nature, timing, and severity of complications from ultrasound-guided percutaneous renal transplant biopsy. Transpl Int 2016;29(02):167-172

41 Chen TK, Gelber AC, Witter FR, Petri M, Fine DM. Renal biopsy in the management of lupus nephritis during pregnancy. Lupus 2015;24(02):147-154

42 Piccoli GB, Daidola G, Attini R, et al. Kidney biopsy in pregnancy: evidence for counselling? A systematic narrative review. BJOG 2013;120(04):412-427

43 Afshin A, Forouzanfar MH, Reitsma MB, et al; GBD 2015 Obesity Collaborators. Health effects of overweight and obesity in 195 countries over 25 years. N Engl J Med 2017;377(01):13-27 